\title{
Review of: "Adipose stem cell niche reprograms the colorectal cancer stem cell metastatic machinery"
}

\author{
Xiongjun Wang
}

Potential competing interests: The author(s) declared that no potential competing interests exist.

Obesity, the excessive accumulation of adipose tissue, has been recognized to be a global health crisis and closely linked to an increased incidence and aggressiveness of cancers. The increased amount of adipose tissue results in higher numbers of adipose stem/stromal cells (ASCs), which has been proven to play a vital role in regulating tumor invasiveness and metastatic potential through several mechanisms, including the increased recruitment of ASCs to the tumor site and increased production of cytokines and growth factors by ASCs and other cells within the tumor stroma.

Emerging evidence indicates that obesity induces alterations in the biologic properties of ASCs, subsequently leading to enhanced tumorigenesis and metastasis of cancer cells. Researchers here have devoted greater effort to defining and understanding the pathophysiological molecular pathways of regulating the biology of the paracrine effect of VAT in the mesenchymal phenotype modulation of CRC cells. They reveal that V-ASCs that prominently present in a primary and metastatic lesion of CRC patients with obesity could release IL-6 and HGF to induce EMT by up-regulating STAT3 phosphorylation and ZEB2 expression, and increase the population of CD44v6+ CRC cells, which are able to colonize the liver and produce CRC metastases. Moreover, CD44v6+ cells recruit V-ASCs to promote tumor aggressiveness feedback by highly expressing and secreting NGF and NFT3. Patients with high liver CRC metastasis affected by obesity are likely due to recruitment from disseminated CD44v6 cells and the migration capacity of V-ASCs. Their data indicate that JAK/STAT pathway and HGF/c-MET pathway may be the therapy targets of obesity associated cancer patients and the microenvironmental cytokines IL6/HGF can be taken as prognostic molecules to predict the tumor progression.

There are still a number of concerns with this study which include the following:

1) In this study, the author basically confirmed that ASCs assist metastatic CRC cells to form stable compartmental microenvironment by secreting IL-6 and HGF, thus promoting CRC. A great concern is that the author did not knock out IL-6 and HGF in ASCs in the colorectal region, so the role of the loss of IL-6 and HGF in the progression of CRC cannot be accurately determined.

2) As is well known that IL6 can be secreted by other cells to promote cancer progression, like immune cells and endothelial cells surrounding or infiltrated in tumor, it is hard to tell about the exact role of the amount of IL-6 secreted by endogenous v-ASCs, though blockade of IL-6 could abolish tumor-prompting effect of artificially injecting excessively exogenous v-ASCs in tumor microenvironment.

3) Another concern is that the overall artificially injection of so many v-ASC cells but not detecting tumor 
progression in lean or fat mice, which need more strictly in vivo experiment to prove the correlation between ASCs and tumor aggression.

4) A paradox is that patients with advanced cancer often die of malnutrition due to high fat consumption, which complicates treatment and adversely affects patient survival. Hence, we should define more carefully the role of white fat in cancer initiation and progression. 